\title{
Access to unregistered drugs in Australia
}

\section{Peter Donovan}

Clinical pharmacologist, General physician and Endocrinologist Royal Brisbane and Women's Hospital Brisbane

\section{Keywords}

Authorised Prescriber Scheme, drug regulation, personal importation, Special Access Scheme, unapproved products

Aust Prescr 2017;40:194-6 https://doi.org/10.18773/ austprescr.2017.062

First published 15 August 2017

\section{SUMMARY}

Drugs can usually only be prescribed for patients if they have been approved by the Therapeutic Goods Administration for inclusion in the Australian Register of Therapeutic Goods.

Unregistered drugs can be obtained through the Special Access Scheme, the Authorised Prescriber Scheme or by personal importation.

Almost any drug can be accessed through these schemes, if it is considered clinically justified.

The use of unregistered drugs should be considered experimental. Written informed consent from the patient is therefore required and any adverse events need to be reported to the Therapeutic Goods Administration.

\section{Introduction}

Before a drug can be supplied to patients in Australia, it must undergo evaluation by the Therapeutic Goods Administration (TGA). If its safety and efficacy, and the quality of its manufacture are satisfactory, it can be included in the Australian Register of Therapeutic Goods. A drug might not be registered in Australia if it is new and there has not yet been time for it to be evaluated by the TGA, or the manufacturer has elected not to have the drug registered in Australia.

In certain circumstances, Australian legislation allows patients to access drugs that are not included in the Australian Register of Therapeutic Goods. Unregistered drugs can be obtained through:

- the Special Access Scheme

- the Authorised Prescriber Scheme

- importation for personal use

- clinical trials.

Almost any drug can be obtained via these schemes, except those for which the manufacture, possession, sale or use are prohibited by law (e.g. illicit drugs).

\section{Special Access Scheme}

The Special Access Scheme allows for the importation and supply of an unregistered drug for an individual patient under the supervision of a medical practitioner, on a case-by-case basis. ${ }^{2}$ This is the most common scheme used by GPs. Circumstances when patient access to an unregistered drug may be appropriate include:

- experimental or investigational products for terminally ill patients

- a drug that has been taken by a patient in a clinical trial, but has not yet received TGA approval
- drugs available overseas, but not marketed in Australia (including when a similar registered product is in short supply in Australia). ${ }^{3}$

Typically, drugs accessed through this scheme are infrequently used and are often for uncommon conditions. The Table shows examples of drugs that have been accessed through the Special Access Scheme at our hospital.

Until July 2017 the Special Access Scheme had two categories of patients. ${ }^{3}$ Category A patients were defined as 'persons who are seriously ill with a condition from which death is reasonably likely to occur within a matter of months, or from which premature death is reasonably likely to occur in the absence of early treatment.' Everyone else was in category B and this was probably the category most used by GPs. Each year there were approximately 40000 category $A$ and 20000 category $B$ applications made to the TGA Following a policy review ${ }^{4}$, a new category was added in July 2017. Category $C$ enables specified types of health professional to prescribe from a list of unapproved drugs that have an established role in the treatment of particular conditions. There are separate lists for drugs, biologicals and devices. Drugs that are not listed in category $C$ are deemed to be higher risk and remain in category $\mathrm{B}$. The TGA has developed an online tool to help prescribers decide which category is appropriate for their patients.

\section{Applications}

To obtain a drug through the Special Access Scheme, an application or notification needs to be made to the TGA. Forms are available from the TGA website (www.tga.gov.au/form/special-access-scheme), and completed forms are emailed (SAS@tga.gov.au) or faxed to the TGA. ${ }^{2}$ 
For category A patients, the prescriber does not need to seek approval from the TGA in advance. A completed 'Special Access Scheme - Category A' form is sent to the supplier (which provides them with the legal authority to supply the product), with a copy of the form to be forwarded to the TGA within four weeks. ${ }^{3}$

For a category B patient, an application to the TGA needs to be completed in advance. An unregistered drug cannot be supplied before the TGA has evaluated and approved the application. ${ }^{3}$

When assessing applications, the TGA takes into consideration whether there is sufficient justification to approve supply of the drug. This includes whether there are registered products already available to treat the patient's condition, whether these products have been tried by the patient in the past, and the seriousness of the patient's condition. ${ }^{3}$ In addition, the TGA considers the degree and quality of evidence to support the drug's efficacy and safety. For example, greater credence is placed on evidence from published randomised trials over individual case reports or expert opinion. ${ }^{3}$ The TGA also considers the relevance of the qualifications of the requesting prescriber in relation to the drug being requested.

For the drugs listed in category $\mathrm{C}$, such as melatonin modified-release tablets for the treatment of sleep disorders, preapproval is not necessary. However, the category $C$ form must be used to notify the TGA within four weeks of supplying the drug.

\section{Supply}

Unregistered drugs may be available from suppliers within Australia, in which case the prescriber (or pharmacy) needs to contact the supplier directly. However, if the drugs are unavailable, the requesting doctor may need to source them from overseas. When this is the case, the prescriber needs to check whether the importation of a drug is controlled by customs regulations. ${ }^{3,5}$ Examples of drugs that are subject to these regulations include: 3,5

- drugs of abuse, for example narcotics, amphetamines, psychotropic substances

- substances that may be considered performance enhancing for athletes, for example anabolic steroids, erythropoietin, growth hormones

- antibiotics.

Drugs subject to these regulations cannot be imported without permission. It is important to note that the import permit and, when required, an import licence for drugs such as narcotics and medical cannabis are obtained through the Office of Drug Control. This process is separate from the TGA's approval. ${ }^{5}$

\section{Table Unregistered products obtained by a} Queensland hospital

\begin{tabular}{ll}
\hline Drug & Condition \\
\hline Special Access Scheme category A & \\
\hline Ceftazadime-avibactam & Multiresistant infection \\
Artesunate & Malaria \\
Diazoxide & Insulinoma \\
\hline
\end{tabular}

\section{Special Access Scheme category B}

$\begin{array}{ll}\text { Midodrine } & \text { Orthostatic hypotension } \\ \text { Rufinamide } & \text { Seizures related to Lennox-Gastaut syndrome } \\ \text { Benzbromarone } & \text { Gout } \\ \text { Mexilitine } & \text { Chronic neuropathic pain }\end{array}$

Special Access Scheme category C

\begin{tabular}{ll}
\hline Paromomycin & Amoebic liver abscess \\
Pristinamycin & Multiresistant infection \\
Tetracycline & Resistant Helicobacter pylori infection \\
Melatonin & Sleep disorders \\
\hline
\end{tabular}

\section{Consent}

The use of unregistered drugs should be considered experimental. It is a condition of the Special Access Scheme that the patient (or their legal guardian) provides written informed consent. ${ }^{3}$ This needs to be provided freely and the patient must understand the nature of their condition (including its natural history) and have appropriate knowledge of the treatment options. Specifically, the patient must be informed about:

- the product not being approved in Australia

- the possible benefits of treatment and any known risks and adverse effects

- the possibility of unknown risks and late adverse effects

- $\quad$ any available alternative treatments using registered products. ${ }^{3}$

It is important for both the patient and prescriber to understand that the Australian Government does not accept responsibility for any adverse consequences of treatment, including any defects in the product related to manufacture. In addition, the prescriber of an unregistered drug is required to report the details of any actual or suspected adverse drug reactions to the TGA within 15 days. ${ }^{3}$ Specific information about the unregistered drug may not be readily available, as the product information for 
the unregistered drug will not be in MIMS and may be limited in the Australian Medicines Handbook. If the drug is registered in another jurisdiction, product information may be available from the US Food and Drug Administration or the European Medicines Agency.

\section{Authorised Prescriber Scheme}

The Authorised Prescriber Scheme also allows access to almost any unregistered drug for particular patient groups. An Authorised Prescriber is a medical practitioner who has been approved by the TGA to prescribe an unregistered drug to a group of patients for a specific indication, without the need for individual TGA approval. ${ }^{6}$ In addition to all the requirements and responsibilities of the prescriber under the Special Access Scheme (including the need for written informed consent from the patient and the reporting of adverse drug events) the doctor requires the endorsement of an ethics committee or relevant specialist college before they can be approved as an Authorised Prescriber. ${ }^{6}$ The use of carboprost as a treatment for postpartum haemorrhage by an obstetrician is a potential example where the Authorised Prescriber Scheme might be appropriate.

The requirement to submit a clinical justification for evaluation by the TGA was removed in July 2017. These reforms also increased the duration of approval for drugs from two to five years. Authorised Prescribers have to report every six months how many patients they are treating with the unregistered drug.

\section{Personal importation}

Personal importation occurs when:

- an individual either brings a therapeutic good into Australia on their person or arranges from within Australia for a therapeutic good to be sent to them from an overseas supplier

- the goods are to be used by that individual or a member of their immediate family and are not sold or supplied to any other person.
For personal importation, the quantity imported on any given occasion cannot exceed three months treatment and no more than 15 months supply can be obtained per year. There are limitations on the type of drugs that can be accessed via personal importation. It is prohibited to import drugs subject to customs regulations (e.g. drugs of abuse, anabolic or androgenic steroids, erythropoietin, growth hormones, gonadotrophins and antibiotics). In these cases, the patient's treating doctor is required to obtain supply under the Special Access Scheme. In addition, with the exception of insulin, the personal importation of an injectable product containing material of human or animal origin is prohibited. For prescription drugs (e.g. Schedule 4 or 8 ), a prescription issued by a medical practitioner registered in Australia (or alternatively, an import licence) is required.

As with accessing non-approved drugs through other means, there can be no guarantees of the quality, safety and efficacy of the imported product. Patients must be prepared to accept the potential harms and benefits of its use. ${ }^{7}$

\section{Clinical trials}

Obtaining a drug as part of a clinical trial requires ethics committee approval and either TGA approval or notification. ${ }^{8}$

\section{Conclusion}

Drugs that are not included in the Australian Register of Therapeutic Goods can be accessed by patients, when clinically justified, via the Special Access Scheme, the Authorised Prescriber Scheme or personal importation. Written informed consent from the patient is required for all unregistered drugs as their use should be considered experimental. More information can be obtained from the TGA website (www.tga.gov.au/accessing-unapproved-products). $<$

\section{Conflict of interest: none declared}

\section{REFERENCES}

1. Therapeutic Goods Administration. Accessing unapproved products. www.tga.gov.au/accessing-unapproved-products [cited 2017 Aug 8]

2. Therapeutic Goods Administration. Special Access Scheme. www.tga.gov.au/form/special-access-scheme [cited 2017 Aug 8]

3. Therapeutic Goods Administration. Special Access Scheme: guidance for health practitioners and sponsors. Version 1.0, July 2017. www.tga.gov.au/special-access-scheme-guidancehealth-practitioners-and-sponsors [cited 2017 Aug 8]

4. Sansom L, Delaat W, Horvath J. Review of medicines and medical devices regulation: recommendations. July 2015 www.health.gov.au/internet/main/publishing.nsf/content/ 8ADFA9CC3204463DCA257D74000EF5A0/\$File/Review\%20 of\%20Medicines\%20and\%20Medical\%20Devices\%20-\%20 Recommendations Accessible.pdf [cited 2017 Aug 8]

5. Australian Government Department of Health. The Office of Drug Control. Importers. 7 December 2016. www.odc.gov.au/ importers [cited 2017 Aug 8]

6. Therapeutic Goods Administration. Authorised Prescriber Scheme: guidance for medical practitioners, human research ethics committees, specialist colleges and sponsors. Version 3.0, July 2017. www.tga.gov.au/authorised-prescriberscheme [cited 2017 Aug 8]

7. Therapeutic Goods Administration. Access to unapproved therapeutic goods: personal importation. October 2004. www.tga.gov.au/publication/personal-import-scheme [cited 2017 Aug 8]

8. Therapeutic Goods Administration. Access to unapproved therapeutic goods: clinical trials in Australia. October 2004. www.tga.gov.au/publication/access-unapproved-therapeuticgoods-clinical-trials-australia [cited 2017 Aug 8] 